IdeAs

Idées d'Amériques

15 | 2020

Eau et gestion de l'eau dans les Amériques

\title{
Susana Draper. Mexico 1968. Constellations of Freedom and Democracy
}

Durham and London, Duke University Press, XVI, 251 pages

\section{Camille Gapenne}

\section{CpenEdition}

\section{Journals}

Édition électronique

URL : http://journals.openedition.org/ideas/7862

DOI : 10.4000/ideas.7862

ISSN : 1950-5701

Éditeur

Institut des Amériques

\section{Référence électronique}

Camille Gapenne, "Susana Draper. Mexico 1968. Constellations of Freedom and Democracy », IdeAs [En ligne], 15 | 2020, mis en ligne le 01 mars 2020, consulté le 24 septembre 2020. URL : http:// journals.openedition.org/ideas/7862 ; DOI : https://doi.org/10.4000/ideas.7862

Ce document a été généré automatiquement le 24 septembre 2020.

\section{c) $\$ \odot$}

IdeAs - Idées d'Amériques est mis à disposition selon les termes de la licence Creative Commons Attribution - Pas d'Utilisation Commerciale - Pas de Modification 4.0 International. 


\title{
Susana Draper. Mexico 1968. Constellations of Freedom and Democracy
}

\author{
Durham and London, Duke University Press, XVI, 251 pages
}

\section{Camille Gapenne}

\section{RÉFÉRENCE}

Susana Draper, Mexico 1968. Constellations of Freedom and Democracy, Durham and London, Duke University Press, XVI, 251 pages, 2018

Prenant comme point de départ des mouvements sociaux récents - tels que 15-M ou Occupy Wall Street - et le cinquantième anniversaire de 1968, Susana Draper cherche, dans cet ouvrage naviguant entre philosophie politique et histoire, à questionner les héritages, la mémoire et les discours dominants sur le mouvement étudiant de 1968 au Mexique. En déplaçant le regard à la marge de l'événement, et au-delà de ses limites chronologiques communément admises, elle met en lumière certaines expériences politiques, des récits dissonants, une histoire occultée de 1968 qui se fraie un chemin jusqu'à l'actualité. Comme son titre l'indique, cet ouvrage donne à voir une constellation d'acteurs et de moments, réunis autour du concept de rencontre, qui gravitent autour d'un événement écrasé par le massacre de Tlatelolco, à la fois dominé par la mémoire de la répression et dépolitisé, rapidement monumentalisé et monopolisé par quelques voix considérées comme légitimes. À la manière de Kristin Ross - largement citée - avec le Mai 68 français, Susana Draper retrace quelques-unes des « vies ultérieures » du 68 mexicain. Au final, l'étude offre au lecteur une réflexion sur la liberté, une liberté qui se pratique dans le politique, et sur la rencontre avec l'autre, qui prend la forme d'une émancipation collective.

Mexico 1968 se divise en quatre grandes parties. La première est chronologiquement contemporaine du mouvement étudiant et de sa répression par le gouvernement de 
Díaz Ordaz. Susana Draper s'intéresse à l'œuvre philosophique et narrative de José Revueltas - philosophe marxiste hétérodoxe - élaborée au contact des étudiants dans la UNAM occupée, puis dans la prison de Lecumberri. Cette première partie, la plus longue et la plus dense, constitue le cœur de l'ouvrage. L'auteure y délimite plusieurs concepts clés qui le jalonnent et l'organisent. Face à l'intensité des événements dont il est à la fois observateur et acteur, José Revueltas cherche à les historiciser en les intégrant dans une temporalité plus large qui permet de penser une autre histoire, souterraine, de surgissements dont le sens déborde leur intense brièveté, et où s'exerce la liberté. Celle-ci, selon le philosophe, ne doit pas être une conquête, un développement téléologique, mais un processus incertain de réinvention permanente, d'ouverture à la critique et à la dissidence, de remise en question des monopoles sur le temps et la parole. Cette "démocratie cognitive" se réalise selon lui dans l'autogestion. L'expérience de l'emprisonnement déplace et approfondit sa réflexion sur la liberté, qu'il défend à travers l'écriture et le langage, en conceptualisant par exemple l'idée d' apando, cellule qui permet également au prisonnier de s'enfermer et de n'être dérangé par personne.

Dans une deuxième partie, la question de la rencontre et des temporalités de 1968 est appliquée à la production et circulation de l'audiovisuel. Susana Draper analyse deux projets cinématographiques qui, facilités par l'apparition du Super 8, ne cherchent pas à diffuser un message militant, mais constituent dans leur conception même une expérience politique et esthétique. La Cooperativa de Cine Marginal, active entre 1971 et 1975, fondée par des étudiants amateurs, avait vocation à réaliser des films (clandestins) dans les usines occupées par les travailleurs. Ceux-ci participaient euxmêmes à la réalisation, faisant de l'usine un théâtre, et du film une expérience collective et d'autogestion. Les projections étaient accompagnées d'un débat et de la distribution de tracts, avec le double objectif de provoquer une prise de conscience et de connecter des luttes dispersées dans le vaste territoire mexicain. Historia de un documento (1971) d'óscar Menéndez, est une production à l'histoire rocambolesque, qui impliqua l'introduction clandestine de caméras dans la prison de Lecumberri, afin que les prisonniers politiques réalisent eux-mêmes des prises de vue. Ici aussi, le projet cinématographique devient lieu de rencontre, expérimentation politique collective, surgissement de la liberté, et il constitue ainsi un prolongement de l'expérience de 68, réprimée et tue.

Les deux derniers chapitres sont dédiés à la voix marginalisée des femmes, malgré leur irruption dans la pratique quotidienne du politique, dans un esprit de radicale égalité et non cantonnée à la cuisine pour les prisonniers (selon une représentation amplement diffusée). Il ne s'agit pas ici de rétablir une sorte de vérité historique quant au rôle des femmes en 1968, mais plutôt d'observer la spécificité de leur regard sur un événement dont la mémoire est presque exclusivement masculine, et d'analyser l'actualité du mouvement de 68 au moyen de textes écrits par des femmes. Susana Draper se penche d'abord sur l'œuvre de la philosophe Fernanda Navarro, en la replaçant dans le contexte de son étroit contact avec le structuraliste Louis Althusser, et plus généralement de la philosophie post-68. Elle développe l'idée de la rencontre comme événement aléatoire, inconfortable et éventuellement conflictuel, et non idéalisé comme harmonie et union.

La dernière partie de l'ouvrage, la plus proche de l'actualité, se centre sur deux témoignages qui, dans un même mouvement, montrent l'hétérogénéité du regard 
féminin, influencé par d'autres facteurs que le genre, et reviennent tous deux à la question de l'expérience de l'emprisonnement, de la rencontre et de la liberté. Roberta Avendaño, membre du Consejo Nacional de Huelga, expose dans De la libertad y el encierro sa méfiance et la difficulté de la cohabitation avec les prisonnières de droit commun venant des classes populaires. À l'inverse, Gladys López Hernández était étudiante du Preparatorio Popular, qui visait à l'intégration dans le Supérieur de jeunes issus des milieux les plus modestes. Malgré les difficultés de l'emprisonnement, elle y trouve un espace où poursuivre les pratiques de solidarité et d'autogestion découvertes pendant le Preparatorio. La rédaction tardive (2013) de son témoignage est justifiée par l'actualité de la demande urgente de démocratie formulée en 1968, toujours lancinante.

L'ouvrage de Susana Draper, particulièrement généreux et dense - qui met en récit de multiples acteurs, moments, approches, concepts et problématiques - constitue finalement une réponse à la préoccupation de José Revueltas quant à la temporalité et à l'historicisation de l'événement: il met en lumière ses résurgences postérieures bien au-delà de ses limites chronologiques, et l'intègre dans une longue histoire de surgissements soudains d'exigence de liberté et de démocratie.

\section{AUTEURS}

\section{CAMILLE GAPENNE}

Universidad de la República (Uruguay) - Université Lyon 2 Lumière (France) 